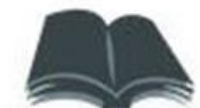 MOLESTO
}

\section{PIOGGIA E ILLUSIONE. UNA CONSIDERAZIONE SU UN LETTORE DI TOLSTOJ}

\author{
Doç. Dr. Raniero Speelman*
}

\section{RIASSUNTO}

Una delle poesie da sempre più amate di D'Annunzio e molto ben documentata è "La pioggia nel pineto" (Alcyone). Accanto all'origine versigliese dove rimanda il titolo, un possibile elemento genetico è offerto da un romanzo di Tolstoj che è conosciuto come "Felicità famigliare" (1859) ma è stato tradotto in francese anche come "Katia" (1886) da August-Henri Blanc de la Nautte Hauterive. Questo testo offre una serie di motivi (silenzio/rumore, rana, illusione, metamorfosi in un essere vegetale) presenti anche nella poesia dannunziana in una descrizione di una pioggia che confronta finalmente dopo anni di incomunicabilità una donna con il marito molto più vecchio. Non è improbabile che il francophone D'Annunzio, avido lettore di romanzi russi, abbia avuto presente questo testo.

Parole chiave: Gabriele D’Annuzio, Tolstoj, Letteratura italiana

Tra le numerose poesie dannunziane, "La pioggia nel pineto" (che fa parte di Alcyone) è probabilmente la più conosciuta e amata dal pubblico. Non è necessario riprodurla per intero nel presente testo, come non ha un vero nucleo diverso dalla descrizione musicale e fonicamente suggestiva di una pioggia in un pineto della Versilia. La nascita della poesia, che potrebbe risalire al 1899 o almeno al 1902, è ben documentata, come si sa anche e più in genere che D'Annunzio si portava dietro nelle escursioni naturalistiche i volumi di dizionari quali il Tommaseo-Bellini in cerca della parola giusta. Il commento esteso dei curatori Anna Maria Andreoli e Niva Lorenzini alla "Pioggia" nell'edizione dei Versi d'amore e di gloria nei Meridiani (1984, d'ora in poi VAG) dà conto dei numerosi motivi che ricollegano questa ad altre poesie della raccolta, come di suoi elementi in comune con la lirica di Shelley, Baudelaire, Pascoli e Petrarca (VAG, p. 1208-1212).

Da quando Aldo Rossi pubblicò la sua lettura 'musicale' della poesia, non sembrano esser rimasti molti incogniti nel componimento poetico del grande abruzzese (Rossi 303-315). A chi scrive è sempre stato un testo caro e suggestivo per il coinvolgimento di una donna per ora nascosta dietro uno dei nomi

\footnotetext{
* Doç. Dr. Raniero Speelman, Utrecht Üniversitesi, Hollanda, ranierospeelman@yahoo.it
} 
ricreati dal poeta, Ermione, cui più tardi dedicò un componimento a parte intitolato "Il nome", ispirato, come pare, alla consultazione del testo Onomasticon di Forcellini (VAG, p. 1213).

Che ruolo ha "la favola bella / che ieri / m'illuse, che oggi t'illude, /o Ermione" (125-128) e che, come si sa, capovolge il rapporto tra i due compagni o amanti dei versi 29-32?

Rileggendo un romanzo breve di Lev Nicolaievic Tolstoj in una edizione francese del 1886 ${ }^{1}$, che reca il titolo di Katia ma è più conosciuto con quello di Felicità matrimoniale (titolo originale: Семейное счастие, traslitterato: Semejnoe sčastie), un testo del 1859 apparso per la prima volta sulla rivista "Russkij vestnik", tradotto da "M. le Comte d'Hauterive" (August-Henri Blanc de la Nautte Hauterive) e pubblicato dalla Librairie académique Didier, Perrin et Cie, Libraires-éditeurs a Parigi (la traduzione, piú volte ristampata, è tuttora in vendita nell'edizione di Climats, ISBN : $2841580032^{2}$ ), mi colpí una scena alla fine del libro. Ho lasciato in quanto segue inalterata la grafia del testo francese.

La trama di Katia è alquanto lineare e si focalizza sul dramma emotivo di una giovane donna diciasettenne che rimane orfana dopo la morte della mamma insieme alla sorella minore e Macha, un'amica di famiglia che ha avuto in precedenza il ruolo di gouvernante. Stranamente, il traduttore ha cambiato i nomi della versione originale, nella quale la narratrice è Macha e la gouvernante si chiama Katia. Ogni tanto viene a far loro visita un vecchio amico del defunto padre, Serge Mikailovitch, che ha almeno 17 anni più di Katia. Il carattere dolce, ponderato e premuroso dell'amico di famiglia, di cui una volta la defunta aveva detto che un uomo come lui sarebbe un marito ideale per Katia, finisce per far innamorarsene la ragazza e malgrado le iniziali riserve dell'uomo che è conscio di quel che potrebbe causare la differenza d'età, i due si sposano e si mettono a convivere con la madre di lui.

Il rapporto si incrina però dopo un soggiorno a Pietroburgo dove Katia scopre il mondo, godendo dell'attenzione prestata alla sua giovinezza e bellezza dall'aristocrazia russa. Il marito, cui non piace la vita di città, non sa reggere bene alla tensione in tal modo nata e si distacca sempre più di lei in una distanza cortese ma - pare - priva d' amore. La nascita di due figli non cambierà questo rapporto; in fondo la maternità non restituisce a Katia l'illusione della possibilità di un rapporto perfetto. Né si lascia sedurre dalle avances di nobiluomini stranieri incontrati durante un soggiorno a Baden Baden che piuttosto la fanno provare la propria vulnerabilità come donna. Una specie di confrontazione si verifica quando durante un'assenza dei figli, marito e moglie si trovano da soli sulla veranda della casa quando si fa sera e scoppia una pioggia. La provocazione di Katia fa sí che i due coniugi parlano finalmente del loro rapporto. Per il

\footnotetext{
${ }^{1}$ Si incontra anche 1888 come data di pubblicazione, data non confermata dal libro originale.

${ }^{2}$ Nell'edizione della Bibliotheque de la Pléjade, la traduzione è invece di Sylvie Lumeau, usata pure per il tascabile della collezione Folio-classique.
} 


\section{MOLESTO: Edebiyat Araştırmaları Dergisi}

marito è andata tutto bene: egli aveva già pensato che la giovane moglie sarebbe rimasta fatalmente affascinata da una vita mondana (Mengaldo).

Nel romanzo, la pioggia funziona da catalizzatore che mette a nudo l'illusorietà. Riportiamo qui sotto il testo che anche il poeta italiano avrebbe potuto leggere nella medesima edizione qui usata ${ }^{3}$. Si sa infatti che D'Annunzio aveva una buona conoscenza della letteratura russa, soprattutto di Dostojevski e Tolstoj, autori che hanno influenzato l'abruzzese al tempo di Giovanni Episcopo e de L'innocente.

Cependant le nuage, en l'absence de tout vent, n'avait cessé de s'abaisser à vue d'oeil sur nos têtes; la nature se faisait de plus en plus calme, plus parfumée, plus immobile: tout à coup une goutte tombe et rebondit, pour ainsi dire, sur la toile de la terrasse, et une autre vient s'émettier sur le blocage du sentier, enfin, avec un bruit de grêle qui s'abat lourdement, commença à éclater une pluie à larges gouttes, rafraîchissante et prenant de la force de moment en moment. Aussitôt rossignols et grenouilles se turent de concert; on n'entendit plus que le bruissement des eaux, bien qu'il fût comme étouffé sous le tapage de la pluie: cependant on le distinguait encore dans l'air, et ily avait aussi je ne sais quel oiseau, sans doute caché sous un ramean de fenilles sèches, qui, non loin de la terrasse, gazouillait sur un rbythme toujours égal ses deux notes monotones. (...)

Une pluie fraiche m'aspergea les cheveux et le cou par jets saccadés. Le nuage, lumineux déjà et devenant à chaque instant plus clair, se fondit en eau sur nous; au bruit régalier de la pluie succéda bientôt celui des gouttes tombant de plus en plus rares du ciel et des fenillages. De nowveau les grenouilles reprirent leurs coassements, de nouveau les rossignols secouèrent leurs ailles et recommencèrent, à se répondre de derrière les touffes bumides, tantôt d'un côté, tantôt de l'autre. Tout redevient serein sous nos yeux. (p. 256-258).

La pioggia purificatrice permette agli sposi di mettersi a parlare del proprio rapporto. Alla "bontà della vita" (Qu'il fait donc bon vivre!, p. 258) vantata dal marito, la moglie contrappone i propri desideri non realizzati.

\footnotetext{
- Oui, jadis cela m'est arrivé à moi aussi, surtout au printemps, me dit-il, comme recueillant ses souvenirs. Oui, mois aussi j'ai passé des nuits entières à former des désirs et des espérances, et de quelle belles nuits que celles-là (...).
}

\footnotetext{
3 Ovviamente, il romanzo è stato anche tradotto in italiano, fino ad oggi almeno 13 volte. V. https://it.wikipedia.org/wiki/Felicit\%C3\%A0_familiare (consultato il 20.2.2018). Se il poeta avesse letto il libro in italiano, ad es. nella prima edizione uscita nel 1895 , forse sotto forma di fenilleton - il sito non ne fornisce ulteriori specificazioni - non farebbe differenza per il presente saggio.
} 


\begin{abstract}
- Ainsi tu ne désires plus rien? Demandai-je.
- Rien d'impossible, répondit-il en devinant mon sentiment. Et toi, vois comme tu as mouillé ta tête, ajouta-t-il en me caressant comme un enfant et passant de nowveau sa main sur mes chevenx: tu es jalouse des fenillages, de l'herbe qui la pluie a monillée; tu voudrais être et l' herbe et les feuilles et la pluie; mais moi je me réjouis seulement en les voyant, comme en voyant tout ce qui est bon, jeune, heureux. (p. 260-261)
\end{abstract}

Il romanzo procede con l'analisi del matrimonio, delle illusioni e dei piaceri che la donna ha avuto, credendo però di perdere l'amore del marito che stava cambiando in qualcos'altro, magari compiacimento di un ordine raggiunto e di una famiglia fondata.

Lungi dal pretendere di aver trovato una fonte sicura della poesia, ho voluto suggerire un momento di lettura che potrebbe aver stimolato la creatività del poeta. È risaputo che la lettura di altri testi in lingua francese, vale a dire le novelle di Guy de Maupassant, ha ispirato non poche Novelle della Pescara. Il racconto "La confession" (quella dei racconti omonimi che inizia con "Tout Véziers-le-Réthel", 1884, raccolto in Toine, ora in Contes et nowvelles II, Paris, Editions de la Pléjade, 1979, pp. 371-378) ha dato la trama del romanzo L'innocente. L'estetica dannunziana è sempre stata molto suscettibile di suggerimenti presi in prestito da autori amati, da Verga ai grandi russi e francesi.

In Katia, il motivo della pioggia impone quasi elementi come la nuvola, le gocce d'acqua, un accelerarsi e poi rallentarsi della violenza della natura ed altri contrasti come quello del silenzio vs. il rumore, le voci di animali (usignuolo, rana), in contrasto tra realtà e illusione. Ciò che forse dovrebbe interessarci di più è l'effetto della pioggia sull'uomo e sulla donna protagonisti del testo. Il motivo della metamorfosi della donna in essere vegetale, è a sua volta forse ispirata al quadro The Tree of Forgiveness di Edward Burne-Jones (https://it.wikipedia.org/wiki/Felicit\% $\% 3 \%$ A0_familiare), già è presente in Tolstoj ("tu voudrais être et l' herbe et les feuilles et la pluie"). Considerati nella loro totalità, gli elementi offrono dei paralleli interessanti che potrebbero aver impressionato il poeta.

\title{
Bibliografia
}

bttps:/ / it.wikipedia.org/wiki/Felicit\%C3\%AO_familiare. 2022018.

Mengaldo, P. V. Una lettura della "Felicità familiare di Tolstòj". Strumenti critici, 2015.

Rossi, Aldo. Stratigrafia di "La pioggia del pineto". Torino: ERI, 1970. 